\title{
Die Kostenneutralität der freipraktizierenden Ärzte stabilisiert sich bei einem schweizweiten Taxpunktwert von 89 Rappen
}

\section{Sitzung des KN-Büros vom 16. Dezember 2004}

\section{U. Stoffel, C. Favrod-Coune}

Auch diesen Monat konnte die Kostenneutralität bei den freipraktizierenden Ärzten ohne besondere Probleme bestimmt werden. Der schweizerische Taxpunktwert stabilisiert sich bei 89 Rappen; ein weit besseres Niveau als dannzumal von den «Experten» vorausgesagt.

Korrespondenz: Backoffice KN-Büro G7 Pflanzschulstrasse 3 CH-8400 Winterthur Tel. 0522351935 Fax 0522351920

E-Mail: KNBueroG7@hin.ch
Die Abweichung der Kostenneutralität gegenüber dem Ausgangswert beträgt knapp 2,34\%. Nach den heute vorgenommenen TPW-Korrekturen liegen die freipraktizierenden Ärzte bei einer Abweichung von 1,72\% und somit klar innerhalb des Korridors.

Inzwischen stehen dem Kostenneutralitätsbüro die Daten von zehn Monaten TARMED zur Verfügung und die strukturellen Divergenzen aus regionaler und tarifbedingter Sicht treten
Tabelle 1 weiterhin der am 1. Januar 2005 festgesetzte TPW. immer deutlicher zutage. Das KN-Büro G7 beschäftigt sich nicht zuletzt im Auftrag der Kantone mit der Analyse und der Aufbereitung obiger Divergenzen. Die politische Würdigung und die Einleitung von daraus folgenden Korrekturmassnahmen bleibt aber den dafür zuständigen Organen vorbehalten.

Weiterhin unerfreulich präsentiert sich der Stand der Kostenneutralitätsmessung bei den Spitälern. Die Vertreter der freipraktizierenden Ärzte haben an der heutigen Sitzung den direktbetroffenen Parteien $\mathrm{H}+$ und santésuisse, aber auch den anwesenden Vertretern des Bundes, unmissverständlich kommuniziert, dass sie nicht gewillt sind, diesen Zustand weiterhin zu tolerieren, und haben insbesondere eine abschlies-

Taxpunktwerte ab 1. Januar 2005 (in Rappen). Stand nach den Beschlüssen des KN-Büros vom 16. Dezember 2004. Die neuen TPW gelten, bis ein neuer TPW festgesetzt wird. Das heisst, erfolgt in einem Monat keine Änderung, so gilt

\begin{tabular}{|c|c|c|c|c|c|c|}
\hline $\begin{array}{l}\text { Kanton/ } \\
\text { KN-Raum }\end{array}$ & Initialwert & ab 1.9. 2004 & ab 1.11.2004 & ab 1.12.2004 & $\begin{array}{l}\text { neu ab } \\
1.1 .2005\end{array}$ & Korrektur \\
\hline Zürich & 97 & 95 & 92 & 92 & 92 & 0 \\
\hline Bern & 89 & 86 & 86 & 84 & 84 & 0 \\
\hline Freiburg & 91 & 91 & 91 & 91 & 88 & -3 \\
\hline Solothurn & 87 & 84 & 84 & 84 & 84 & 0 \\
\hline Basel-Stadt & 93 & 93 & 93 & 91 & 89 & -2 \\
\hline Basel-Landschaft & 97 & 97 & 94 & 92 & 92 & 0 \\
\hline Graubünden & 84 & 78 & 78 & 78 & 78 & 0 \\
\hline Aargau & 89 & 89 & 89 & 89 & 87 & -2 \\
\hline Tessin & 91 & 97 & 97 & 97 & 97 & 0 \\
\hline Waadt & 92 & 96 & 98 & 98 & 98 & 0 \\
\hline Wallis & 78 & 78 & 78 & 78 & 78 & 0 \\
\hline Neuenburg & 94 & 89 & 89 & 89 & 89 & 0 \\
\hline Genf* & 98 & 98 & 98 & 98 & 98 & 0 \\
\hline Jura & 95 & 95 & 97 & 97 & 97 & 0 \\
\hline Zentral-CH & 86 & 83 & 80 & 80 & 80 & 0 \\
\hline Ost-CH & 85 & 82 & 82 & 82 & 82 & 0 \\
\hline
\end{tabular}


sende, für die freie Ärzteschaft verträgliche Lösung per 1. Februar 2005 gefordert. Es ist unbestritten, dass den Spitälern aus dieser unerfreulichen Situation nicht auch noch Vorteile erwachsen dürfen.
Auf Antrag des PVB wurde auch eine leichte Anpassung der seit neun Monaten geltenden Notmassnahmen Radiologie beschlossen.

\section{Neutralité des coûts chez les médecins} en pratique privée: situation stabilisée avec une valeur moyenne du point de 89 centimes au niveau suisse

\section{7e séance du Bureau de la neutralité des coûts du 16 décembre 2004}

\section{U. Stoffel, C. Favrod-Coune}

Ce mois encore, les données concernant la neutralité des coûts chez les médecins libres praticiens ont pu être établies sans difficulté particulière. La valeur moyenne du point tarifaire sur le plan suisse se stabilise à 89 centimes, un niveau bien meilleur que ce qu'avaient prédit les «experts» à l'époque.
La neutralité des coûts s'écarte actuellement de près de $2,34 \%$ de la valeur initiale de référence. Suite aux adaptations de la valeur du point tarifaire effectuées aujourd'hui, les médecins en
Correspondance:

Service technique Bureau NC G7

Pflanzschulstrasse 3

CH-8400 Winterthur

Tél. 0522351935

Fax 0522351920

E-mail: KNBueroG7@hin.ch

Tableau 1

Valeurs du point tarifaire (en centimes) dès le 1er janvier 2005. Situation selon les décisions prises le 16 décembre 2004 par le Bureau de la neutralité des coûts. Les nouvelles VPT restent en vigueur jusqu'à la prochaine modification de la valeur du point. En d'autres termes, lorsque aucune adaptation n'intervient au début d'un mois, la VPT fixée au $1^{\text {er }}$ janvier 2005 demeure valable.

\begin{tabular}{|c|c|c|c|c|c|c|}
\hline Canton/Zone & valeur initiale & $\begin{array}{l}\text { dès le } \\
1.9 .2004\end{array}$ & $\begin{array}{l}\text { dès le } \\
1.11 .2004\end{array}$ & $\begin{array}{l}\text { dès le } \\
1.12 .2004\end{array}$ & $\begin{array}{l}\text { nouvelle dès le } \\
1.1 .2005\end{array}$ & correction \\
\hline Zurich & 97 & 95 & 92 & 92 & 92 & 0 \\
\hline Berne & 89 & 86 & 86 & 84 & 84 & 0 \\
\hline Fribourg & 91 & 91 & 91 & 91 & 88 & -3 \\
\hline Soleure & 87 & 84 & 84 & 84 & 84 & 0 \\
\hline Bâle-Ville & 93 & 93 & 93 & 91 & 89 & -2 \\
\hline Bâle-Campagne & 97 & 97 & 94 & 92 & 92 & 0 \\
\hline Grisons & 84 & 78 & 78 & 78 & 78 & 0 \\
\hline Argovie & 89 & 89 & 89 & 89 & 87 & -2 \\
\hline Tessin & 91 & 97 & 97 & 97 & 97 & 0 \\
\hline Vaud & 92 & 96 & 98 & 98 & 98 & 0 \\
\hline Valais & 78 & 78 & 78 & 78 & 78 & 0 \\
\hline Neuchâtel & 94 & 89 & 89 & 89 & 89 & 0 \\
\hline Genève* & 98 & 98 & 98 & 98 & 98 & 0 \\
\hline Jura & 95 & 95 & 97 & 97 & 97 & 0 \\
\hline Suisse centrale & 86 & 83 & 80 & 80 & 80 & 0 \\
\hline Suisse orientale & 85 & 82 & 82 & 82 & 82 & 0 \\
\hline
\end{tabular}


pratique privée affichent un écart de 1,72\% et respectent ainsi clairement la marge de tolérance fixée $(2 \%)$.

Le Bureau de la neutralité des coûts dispose désormais de données couvrant dix mois de facturation sous le TARMED, ce qui permet de mieux cerner les divergences structurelles d'ordre régional et tarifaire qui se font jour. Le Bureau de la neutralité des coûts du G7 se charge - notamment sur mandat des cantons - d'analyser et de quantifier ces divergences. L'évaluation politique des différences constatées et la mise en route de mesures de correction incombent en revanche aux organes concernés.

La situation est moins réjouissante en ce qui concerne l'appréciation de la neutralité des coûts dans le secteur hospitalier. Lors de la séance de ce jour, les représentants des médecins libres praticiens ont signifié sans équivoque aux parties directement concernées, à savoir $\mathrm{H}+$ et santésuisse, mais également aux délégués de la Confédération présents sur place, qu'ils ne sont pas prêts à continuer de tolérer un tel état de choses. Ils ont notamment exigé pour le $1^{\text {er }}$ février 2005 une solution définitive qui soit acceptable pour les médecins exerçant à leur propre compte. Personne ne conteste que les hôpitaux ne devraient pas, en plus, retirer des avantages de cette malencontreuse situation.

A la demande du bureau paritaire de préexamen, il a en outre été procédé à une légère adaptation des mesures d'urgence en vigueur depuis neuf mois dans le domaine de la radiologie.

\title{
FMH Tarifdienst
}

\author{
Neue Adresse \\ Der FMH Tarifdienst ist seit 1. Januar 2005 unter folgender Adresse erreichbar: \\ FMH Tarifdienst \\ Gösgerstrasse 8 \\ 4600 Olten \\ Tel. 0622879696 \\ Fax 0622879690 \\ E-Mail: fmhtarif@hin.ch (unverändert) \\ Das ganze Team freut sich, Sie auch im neuen Jahr begrüssen zu dürfen.
}

\section{Service tarifaire de la FMH}

\section{Nouvelle adresse}

Dès le $1^{\text {er }}$ janvier 2005, le Service tarifaire de la FMH dispose d'une nouvelle adresse:

Service tarifaire de la FMH

Gösgerstrasse 8

4600 Olten

tél. 0622879696

fax 0622879690

e-mail: fmhtarif@hin.ch (inchangé)

Toute l'équipe se réjouit de pouvoir vous conseiller en 2005. 